\title{
Social modifications of the multiple birth effect on IQ and body size: a population-based study of young adult males
}

\section{Silventoinen, Karri}

2013

Silventoinen, K, Myrskylä , M , Tynelius , P , Yokoyama , Y \& Rasmussen , F 2013 , ' Social modifications of the multiple birth effect on IQ and body size: a population-based study of young adult males ' , Paediatric and perinatal epidemiology , vol. 27 , no. 4 , pp. 380-387 . https://doi.org/10.1111/pp

http://hdl.handle.net/10138/234029

https://doi.org/10.1111/ppe.12054

acceptedVersion

Downloaded from Helda, University of Helsinki institutional repository.

This is an electronic reprint of the original article.

This reprint may differ from the original in pagination and typographic detail.

Please cite the original version. 
Social modifications of the multiple birth effect on IQ and body size:

a population-based study of young adult males

$$
\begin{aligned}
& \text { Karri Silventoinen }^{\mathrm{a}} \\
& \text { Mikko Myrskyläb }^{\mathrm{b}} \\
& \text { Per Tynelius } \\
& \text { Yoshie Yokoyama }^{\mathrm{d}} \\
& \text { Finn Rasmussen }^{\mathrm{c}}
\end{aligned}
$$

aPopulation Research Unit, Department of Social Research, University of Helsinki, Helsinki, Finland

${ }^{b}$ Max Planck Research Group Lifecourse Dynamics and Demographic Change, Max Planck Institute for Demographic Research, Rostock, Germany

${ }^{\mathrm{c} C h i l d ~ a n d ~ A d o l e s c e n t ~ P u b l i c ~ H e a l t h ~ E p i d e m i o l o g y, ~ D e p a r t m e n t ~ o f ~ P u b l i c ~ H e a l t h ~}$ Sciences, Karolinska Institutet, Stockholm, Sweden

${ }^{\mathrm{d}}$ Department of Community Health Nursing, Osaka City University, Osaka, Japan

Corresponding author

Karri Silventoinen

University of Helsinki

Population Research Unit

Department of Social Research

P.O. Box 18

FIN-00014 University of Helsinki

Finland

Tel: +358919123083

Fax: +358919123967

GSM: +358400-620726

email: karri.silventoinen@helsinki.fi 
Abstract

Background: Twins and triplets fall behind singletons in cognitive and physical development. We analyzed whether these differences are modified by family social position.

Methods: IQ, height, body mass index (BMI) and elbow flexion, handgrip and knee extension strength were measured in Swedish men born between 1951 and 1976 during the conscription examination at 17 to 20 years of age. Information from Swedish registers on all siblings and parental socioeconomic position were linked to these measures. Together the measures were available for 1,140,329 singletons, 19,755 twins and 207 triplets. The data were analyzed using regression analyses and fixed-effect models which compare twins and triplets to their singleton brothers.

Results: Twins and triplets had slightly lower IQ, height, BMI and muscle strength than did singletons both at the population level as well as when compared to their singleton brothers. Although the differences between twins and triplets were small, they were largest in families with lower socioeconomic position. We also found larger differences in IQ among the sons of mothers born before 1940.

Conclusions: Twins and triplets fall slightly behind singletons in cognitive and physical development, even in early adulthood, but twins and triplets show no major differences. These differences are largest in families in a low socioeconomic position. Our results underscore the importance of the post-natal environment in the 
development of twins and triplets and suggest that their parents, especially those in a low socioeconomic position, need special support.

Key words: IQ, body size, twins, triplets, social class 
The long-term consequences of multiple births on child development have become an important pediatric issue in recent decades as the number of multiple births has dramatically increased due to the growing use of in vitro fertilization. ${ }^{1}$ A metaanalysis of 16 studies found an overall twin-singleton difference in IQ of 4.2 points, a smaller difference in recent birth cohorts ( 0.5 IQ points) than in earlier birth cohorts (5.1 IQ points). ${ }^{2}$ Two large studies based on school records in the UK in $2004^{3}$ and in the Netherlands in 1994-2003, ${ }^{4}$ which reported no difference in IQ between twins and singletons in mid-childhood, also gave evidence of the diminishing IQ difference between twins and singletons. With regard to physical development, evidence suggests that twins are lighter than singletons in late adolescence ${ }^{5}$ despite their rapid catch-up growth in early childhood. ${ }^{6}$ Studies of the development of triplets are rare, but reports from Japan suggest that triplets fall behind singletons in their motor development in early childhood ${ }^{7}$ as well as growth in height ${ }^{8}$ and weight ${ }^{9}$ in midchildhood.

An important public health issue is whether a good post-natal environment could attenuate the negative effects of multiple pregnancies on child development as suggested by the diminishing difference in IQ between twins and singletons in recent birth cohorts. ${ }^{2}$ Thus far, however, this question has not been studied systematically in physical and cognitive development, and studies of triplets are rare. We therefore decided to analyze differences in IQ, height, body mass index (BMI), and muscle strength in early adulthood between twins, triplets and singletons in a large and representative cohort of Swedish men and to analyze whether they are modified by birth cohort and parental socio-economic position (SEP). Because we had information on the singleton brothers of twins and triplets, we were also able to account for all familial effects in our analyses. 
Participants and methods

IQ, height, and weight as well as elbow flexion, handgrip, and knee extension strength were measured during the conscription examination between 1969 and 1994 . In these cohorts, the conscription examination preceded military service and was compulsory by law for all healthy male Swedish citizens; only those with a severe handicap or chronic disease verified by a physician were exempt. The median age at the time of the conscription examination was 18.2 years. To make the data more homogeneous for the measurement age, we excluded those who were younger than 17 or older than 20 years at the time of the examination $(19,010$ men), since they comprised only a small fraction of the entire study population (1.6\%). Information on the conscripts' mothers, fathers, and all siblings was linked to data from the Swedish Multi-Generation Register through unique personal identification numbers. Our final study cohort comprised 1,140,329 singletons, 19,755 twins, and 207 triplets born between 1951 and 1976. The study was approved by the Ethical Review Board, Stockholm, Sweden.

A combined IQ score was derived as a summary score from four subtests measuring logical, verbal, spatial, and technical capabilities. The global IQ was standardized to yield a Gaussian distributed score from 1 to 9. The Cronbach $\alpha$ for the global IQ score was 0.83 , suggesting the high internal consistency of this measure. To make the results more comparable to those of previous IQ studies, we transformed the IQ score to yield a mean of 100 and a standard deviation of 15 points. The Swedish Army did not reveal to us the measurement protocol of the muscle strength tests. However, no systematic differences were evident in the mean values of the measures 
between conscription offices, suggesting the use of a uniform protocol. The values of elbow flexion, handgrip and knee extension strength in these data were also close to those in a previous study of 31- to 35-year-old Finnish men. ${ }^{10}$

Height and weight were measured in underwear and served to calculate BMI $\left(\mathrm{kg} / \mathrm{m}^{2}\right)$. Height and muscle strength values were approximately normally distributed, but to normalize the distribution for BMI, we had to make a logarithmic transformation. A small number of measures were rejected as outliers for height $(0.2 \% ; 160-200 \mathrm{~cm})$, BMI $\left(0.5 \% ; 16-35 \mathrm{~kg} / \mathrm{m}^{2}\right)$, elbow flexion $(0.13 \% ; 150-750$ newtons), handgrip $(0.13 \% ; 150-990$ newtons $)$, and knee extension strength $(0.04 \%$; 180-999 newtons) by replacing them with missing values because they fell outside the limits of the distributions. These values can be measurement or coding errors or correct values, but influenced by other genetic, idiopathic or environmental factors than those with effects falling within the normal range of variation.

The SEP of the parents, based on occupation, was derived from the Swedish Population and Housing Censuses and linked to the data through personal identification numbers. This information was extracted from the 1990 census and, if unavailable or coded as unclassified, was extracted from the 1980, 1970, or 1960 census in that order. SEP was classified into seven classes: higher-level non-manuals, mid-level non-manuals, lower-level non-manuals, farmers, skilled workers, unskilled workers, and unclassified. We classified those families with a mother or father in the higher- or mid-level non-manual positions as higher SEP families ( $40 \%$ of the conscripts) and others as lower SEP families. Further, we classified the mother's birth year (from 1900 to 1961) as before 1940 (58\% of conscripts) and 1940 or after. We used the mother's birth year instead of the birth year of the conscript because some families may have brothers who belong to different birth cohorts and would thus be 
split if we were to use the conscript's birth year. Using the mother's birth year ensures that the same families appear in all analyses.

We began the analyses using ordinary multiple regression analysis. Separate models were used for IQ, height, $\log$ BMI, and the three muscle strength measures. LogBMI values were multiplied by 100 and can thus be interpreted as percent changes. The probability of dizygotic twin pregnancies increases with maternal aging. ${ }^{11}$ Thus we adjusted the results for the age of the mother at the time of delivery in addition to the birth year of the conscript, age at conscription, and conscription office. Swedish young men are required to enlist at the conscription office closest to their residence. Thus, adjusting the results for the conscription office adjusts the results for possible geographic variation in Sweden. We also found that both the mother's and father's SEP was associated with multiple birth $(\mathrm{p}<0.0001)$, we so decided to adjust the results for maternal and paternal SEP also. In addition, we adjusted the results for the number of siblings in order to account for any possible negative effect of the larger family size on the postnatal resources of the family.

We continued the analyses by using a fixed-effect regression model. In this model, a dummy variable is fitted for each set of siblings with the same mother. Thus, maternal half-brothers were also included because they are likely to have grown up in the same family. The model compares brothers born to the same mother and removes the confounding influences of all fixed observed and unobserved genetic and social characteristics that the brothers share. All models were estimated using Stata/SE 11.2 statistical software (StataCorp, College Station, Texas, USA). We used Stata's cluster option to take into account the effect of the non-random sample design on the standard errors. 
Results

Table 1 presents the unadjusted means and standard deviations (SD) for IQ and anthropometric traits. Twins had lower IQ by 2.6 points, shorter stature by 0.6 $\mathrm{cm}$, lower BMI by $0.6 \mathrm{~kg} / \mathrm{m}^{2}$, and less muscle strength by 11 to 21 newtons than did singletons. In triplets, the differences were largely similar to those observed in twins, who had lower IQ by 2.1 points, shorter stature by $0.7 \mathrm{~cm}$, lower BMI by $0.9 \mathrm{~kg} / \mathrm{m}^{2}$ and less muscle strength by 16 to 23 newtons than did singletons. No systematic differences in the SDs of IQ or the anthropometric traits were observed between singletons, twins, and triplets. Further, we found no differences in conscription age and birth year, but the maternal age was 1.6 years higher in twins, and 2.2 years higher in triplets, than in singletons.

Table 2 presents the results of ordinary regression analyses adjusted for birth year, conscription office, conscription age, mother's age at the time of delivery, number of siblings, and maternal and paternal SEP. Twins and triplets had lower IQ and shorter height than did singletons, but the differences were not statistically significant in triplets. Both total body mass measured as $\operatorname{logBMI}$ and muscle mass approximated by muscle strength were lower for twins and triplets than for singletons. When we studied the differences between twins and triplets by testing the equality of the parameter estimates, we found that these differences were not statistically significant $(\mathrm{p}=0.22-0.98)$.

We found statistically significant interactions with parental SEP or maternal birth cohort for IQ, $\log B M I$, and muscle strength tests, but not for height. The difference in IQ was greater in lower SEP families $(\mathrm{p}=0.013)$ and in the sons of mothers born before $1940(\mathrm{p}=0.0008)$. LogBMI and all three muscle strength 
measures also showed statistically significant interaction for parental SEP $(p=$ 0.0001-0.02). In the lower SEP families, the differences in $\operatorname{logBMI}$ and strength measures of twins and triplets, when compared to those of singletons, were larger than in the higher SEP families; the only exception was elbow flexion strength in triplets. Statistically significant interaction for the mother's birth year for the anthropometric measures was found for elbow flexion $(\mathrm{p}=0.01)$ and knee extension strength $(\mathrm{p}=$ 0.03). However, these results were somewhat mixed, and generally larger differences were found for mothers born in 1940 or later.

Table 3 presents the corresponding analyses using the fixed-effect model. These models compare sons born to the same mother and thus effectively adjusts the results for all maternal factors fixed between different pregnancies of the same women. We also adjusted the results for birth year, conscription office, and conscription age because these factors show variation between brothers. The results were largely similar to those of the regression model noted above. Twins had lower IQ, shorter stature, lower $\log B M I$, and less muscle strength. The results for triplets were largely similar, but because of the lower statistical power of these analyses, the results were not statistically significant. We found no statistically significant differences between twins and triplets $(\mathrm{p}=0.22-0.88)$. Further, we found no statistically significant interactions for parental SEP or birth year of the mother, which may also indicate the lower statistical power of these analyses.

\section{Discussion}

Our results, which showed lower IQ in twins and triplets than in singletons, are in line with those of previous studies reporting similar differences between twins 
and singletons. A recent meta-analysis found that this difference was 4.1 points, whereas in this cohort the difference was 2.6 points. ${ }^{2}$ Comparing the IQ of twins to their singleton brothers, thereby taking into account all differences between families, revealed similar differences. To our knowledge, this is the first study to report an IQ difference between triplets and singletons. Even though triplets have slightly lower IQ than singletons, this difference seems to be no larger than in twins. Thus, even when triplets have lower birth weight and lower gestational age than twins, ${ }^{12}$ they do not seem to experience more problems in their cognitive development. It is worth noting that the latest-born participants of this study were born in 1976. Thus, all conceptions were natural since in vitro fertilization became available in Sweden in the early 1980s. ${ }^{13}$ The results of more recent studies, which compared IQ in twins and singletons and found no differences, ${ }^{3,4}$ may be biased due to higher SEP of families using in vitro fertilization, which carries a higher probability of dizygotic twins. ${ }^{1}$ Interestingly, another Swedish twin study based on later birth cohorts (1973-1981) found that twins showed somewhat higher academic achievement despite their slightly lower IQ. ${ }^{14}$ This result suggests that social factors, such as support from the co-twin, may offset for twins' disadvantage in IQ.

In addition to the difference in IQ, we found that twins and triplets were shorter, lighter, and had weaker muscle strength than did singletons. Although we previously have reported the unadjusted means of anthropometric traits in twins and singletons as part of another study, ${ }^{15}$ the present study also reports on triplets and confirms that these results hold true when compared to the singleton brothers of twins and triplets. These findings are in line with a previous Finnish study which found that twins had lower BMI in late adolescence than did singletons. ${ }^{5}$ These results show that even in adulthood, catch-up growth during childhood does not completely compensate 
for the effect of small birth size of twins. ${ }^{6}$ Triplets were shorter, had lower BMI, and weaker muscle strength than did twins, but these differences were relatively small and not statistically significant. Thus physical development in triplets seems to be largely similar to that observed in twins.

Our most novel results concern socioeconomic differences in the cognitive and physical development of twins and triplets when compared to that of singletons. We found that in families with higher SEP, differences in both IQ and the physical characteristics of twins and triplets, when compared to those of singletons, were smaller than in families with lower SEP. This suggests that families with higher SEP have more resources, both material and social, to support their children and thus compensate for the negative consequences of the suboptimal pre-natal environment related to multiple birth. Another explanation is that some of these differences in the low SEP families originate in post-natal life when the parents must cope with the demands of two or three children of the same age simultaneously. To take this effect into account, we adjusted the results for the number of siblings. This adjustment decreased the differences in the IQ and height of twins and triplets from those of singletons suggesting that post-natal resources affect these differences (the unadjusted results, though not shown here, are available from the corresponding author). However, caring for two or three children of the same age is more demanding than caring for children of different ages. Thus, adjusting the results only for family size does not necessarily account for all extra post-natal stress for twins and triplets.

When we stratified the analyses by the mother's birth cohort, we found that differences in the IQ of twins and triplets, when compared to that of singletons, were larger among the sons of mothers born before 1940 than among those of mothers born in or after 1940. However, in contrast to the results stratified by parental SEP, we saw 
no similar interaction effects for the anthropometric measures. We used the mother's birth year instead of the participant's own birth year to ensure that the same data are used in all fixed-effect analyses comparing twins and triplets to their singleton brothers. They are strongly correlated, however, and the median birth year of the sons of mothers born before 1940 was 1958, whereas it was 1971 for the sons of mothers born in or after 1940. These results for IQ are likely to reflect differences in the postnatal environment. From 1958 to 1971, the standard of living in Sweden improved dramatically. For example, the under-five-year mortality rate decreased from 19.18 to 13.02 per 1000 live births, ${ }^{16}$ while at the same time gross domestic product per capita increased 1.6 fold based on reference prices from $2000 .{ }^{17}$ Correspondingly a previous meta-analysis found that IQ differences between twins and singletons were smaller in recent cohorts than in earlier ones. ${ }^{2}$ In this meta-analysis, the difference in the effect sizes between the birth cohorts was larger ( 0.5 vs. 5.1 IQ points) than in our study (1.9 vs. 2.4 IQ points). However, the time difference between the birth cohorts was also much smaller in our study, so these results are quite comparable. Advantages of our data are that our results are based on the same population and the data were collected using the same measurement protocol. An open question, however, is why no similar differences in physical development were found even though they were evident when stratifying the results for parental SEP.

Our data have both important strengths and limitations. The main strength of our data is that our IQ and anthropometric measures, including muscle strength test results, cover a large number of singletons, twins, and triplets measured using a uniform protocol in early adulthood within very narrow age limits. The large sample size is necessary for the stratified analyses and when studying triplets, as triplet pregnancies are rare. We were also able to compare twins and triplets to their 
singleton brothers and thus effectively take into account any shared genetic and social factors. Because the conscription examination was mandatory for all healthy Swedish men in our birth cohorts, our data are not prone to self-selection, which could otherwise bias the results. Finally the information on paternal and maternal SEP was register-based and thus more reliable than if we had used self-reported data.

A major limitation of our data is that for these birth cohorts, we had no information on birth-related factors. It is well known that twins and, to an even larger degree, triplets have lower birth weight and earlier gestational age than do singletons. ${ }^{12}$ Previous studies have also shown that birth size affects both physical ${ }^{8,9}$ and cognitive development ${ }^{18}$ in twins and triplets. However, when comparing twins and triplets to singletons, taking into account the effect of gestational age or birth size is problematic. For twins and triplets, growth retardation occurs largely during the third trimester of pregnancy and is a natural consequence of the physical constraints of the uterus. ${ }^{19}$ However, low birth weight and early gestational age in singletons can result because of multiple maternal factors varying from disease to smoking and low SEP. ${ }^{20}$ Thus, low birth weight and low gestational age is expected to show different associations with the development of singletons when compared to twins and triplets. We found evidence for this in Japanese children, since very low birth weight triplets were ahead of very low birth weight singletons in motor development in early childhood. ${ }^{7}$ Further, we used no information on zygosity because it was available only for part of the cohort, and stratification of the results by zygosity would have decreased the statistical power in the analysis stratified by parental SEP and maternal birth cohort. However, in our previous study in which we used self-reported zygosity and some of the same data as in this study, we found no major differences in height, BMI, or muscle strength between monozygotic and dizygotic twins. ${ }^{15}$ The fact that we 
used no conventional IQ test, but our IQ measures were based on a test developed by the Swedish Army, could also be regarded as a limitation. In our previous study, however, we found that this test, for example, predicts mortality ${ }^{21}$ and is associated with the participant's own and parental education and $\mathrm{SEP}^{22}$ in a way similar to that of other studies that use conventional IQ tests. Further, the IQ measure is based on four sub-tests measuring logical, verbal, spatial, and technical capabilities and consequently is not limited to a single domain of cognitive ability. Thus, the test used in this study is likely to measure general cognitive ability as measured by the other IQ tests also. It is also worth noting that men with a severe handicap or long-term disease were exempted from the conscription examination. The probability of severe birth defects, such as cerebral palsy, is much higher in multiple than in singleton births. ${ }^{23}$ Thus, our results can be generalized only to healthy twins and triplets. Finally, because we had information only on males, we cannot directly generalize the results to females.

To conclude, twins and triplets have somewhat suboptimal cognitive and physical development compared to that of singletons. This difference was small, however, and triplets showed no greater cognitive or physical delay than did twins. It is worth noting that the lag in the cognitive and physical development of twins and triplets was greatest in lower SEP families, which probably have fewer resources than do higher SEP families. Thus, supporting families with twins and triplets is vital in minimizing the possible harmful effects of multiple birth on the further development of the offspring.

\section{References}


(1) Imaizumi Y. A comparative study of zygotic twinning and triplet rates in eight countries, 1972-1999. Journal Biosocial Science 2003;35:287-302.

(2) Voracek M, Haubner T. Twin-singleton differences in intelligence: a metaanalysis. Psychological Reports 2008;102:951-962.

(3) Calvin C, Fernandes C, Smith P, Visscher PM, Deary IJ. Is there still a cognitive cost of being a twin in the UK? Intelligence 2009;37:243-248.

(4) Webbink D, Posthuma D, Boomsma DI, de Geus EJC, Visscher PM. Do twins have lower cognitive ability than singletons? Intelligence 2008;36:539-547.

(5) Pietiläinen KH, Kaprio J, Räsänen M, Rissanen A, Rose RJ. Genetic and environmental influences on the tracking of body size from birth to early adulthood. Obesity Research 2002;10:875-884.

(6) van Dommelen P, de Gunst M, van der Vaart A, van Buuren S, Boomsma D. Growth references for height, weight and body mass index of twins aged 0-2.5 years. Acta Paediatrica 2008;97:1099-1104.

(7) Yokoyama Y, Sugimoto M, Miyake Y, Sono J, Mizukami K, Kaprio J, et al. Motor development of triplets: a Japanese prospective cohort study. Twin Research and Human Genetics 2011;14:185-191.

(8) Yokoyama Y, Sugimoto M, Pitkäniemi J, Kaprio J, Silventoinen K. Height growth of triplets from birth to 12 years of age in Japan. Twin Research and Human Genetics 2011;14:468-475. 
(9) Yokoyama Y, Pitkäniemi J, Kaprio J, Silventoinen K. Weight growth of triplet infants from birth to twelve years of age. Twin Research and Human Genetics 2012;15:672-679.

(10) Viitasalo JT, Era P, Leskinen AL, Heikkinen E. Muscular stregth profiles and anthropometry in random samples of men aged 31-35, 51-55 and 71-75 years. Ergonomics 1985;28:1563-1574.

(11) Macfarlane A, Blondel B. Demographic trends in Western European countries. In: Blickstein I, Keith LG, editors. Multiple Pregnancy: Epidemiology, Gestation and Perinatal Outcome. Second ed. London and New York: Tylor \& Francis; 2005. p. 1121.

(12) Luke B, Brown MB, Hediger ML, Misiunas RB, Anderson E. Perinatal and early childhood outcomes of twins versus triplets. Twin Research and Human Genetics 2006;9:81-88.

(13) Kallen B, Finnstrom O, Nygren KG, Olausson PO. Temporal trends in multiple births after in vitro fertilisation in Sweden, 1982-2001: a register study. BMJ 2005(7513):382-383.

(14) Hjern A, Ekeus C, Rasmussen F, Lindblad F. Educational achievement and vocational career in twins - a Swedish national cohort study. Acta Paediatrica 2012;101:591-596.

(15) Silventoinen K, Magnusson PK, Tynelius P, Kaprio J, Rasmussen F. Heritability of body size and muscle strength in young adulthood: a study of one million Swedish men. Genetic Epidemiology 2008;32:341-349. 
(16) Gapminder Foundation. Under-five mortality rate (per 1,000 live births). 2012; Available at: www.gapminder.org.

(17) Portal for Historical Statistics. GDP per capita in Sweden 1720-2000 in the reference prices of year 2000. 2012; Available at: http://www.historicalstatistics.org/.

(18) Edmonds CJ, Isaacs EB, Cole TJ, Rogers MH, Lanigan J, Singhal A, et al. The effect of intrauterine growth on verbal IQ scores in childhood: a study of monozygotic twins. Pediatrics 2010;126:e1095-101.

(19) Malinowska K, Antosik A, Balcerczak M. The uterine capacity measured by the total twin birth weight and duration of pregnancy. Acta Geneticae Medicae et Gemellologiae 1998;47:183-190.

(20) Valero De Bernabe J, Soriano T, Albaladejo R, Juarranz M, Calle ME, Martinez D, et al. Risk factors for low birth weight: a review. European Journal of Obstetrics \& Gynecology and Reproductive Biology 2004;116:3-15.

(21) Batty GD, Wennerstad KM, Smith GD, Gunnell D, Deary IJ, Tynelius P, Rasmussen F. IQ in early adulthood and mortality by middle age: cohort study of 1 million Swedish men. Epidemiology 2009;20:100-9.

(22) Silventoinen K, Modig-Wennerstad K, Tynelius P, Rasmussen F. Association between intelligence and coronary heart disease mortality: a population-based cohort study of 682361 Swedish men. Eur J Cardiovasc Prev Rehabil 2007;14:555-60.

(23) Glinianaia SV, Jarvis S, Topp M, Guillem P, Platt MJ, Pearce MS, et al. Intrauterine growth and cerebral palsy in twins: a European multicenter study. Twin Research and Human Genetics 2006;9:460-466. 
Table 1. Means and standard deviations (SD) of IQ and anthropometric measures for singletons, twins and triplets.

\begin{tabular}{|c|c|c|c|c|c|c|}
\hline & Single & ns & Tw & & Trip & \\
\hline & Mean & SD & Mean & SD & Mean & SD \\
\hline IQ (point) & 100.0 & 14.99 & 97.4 & 15.19 & 97.9 & 14.83 \\
\hline Height $(\mathrm{cm})$ & 179.2 & 6.41 & 178.6 & 6.50 & 178.5 & 6.33 \\
\hline Body mass index $\left(\mathrm{kg} / \mathrm{m}^{2}\right)$ & 21.7 & 2.79 & 21.1 & 2.55 & 20.8 & 2.41 \\
\hline Elbow flexion strength (newton) & 387 & 83.1 & 375 & 81.3 & 366 & 81.5 \\
\hline Hand grip strength (newton) & 616 & 96.8 & 605 & 95.3 & 600 & 90.9 \\
\hline Knee extension strength (newton) & 569 & 117.2 & 548 & 113.0 & 546 & 110.1 \\
\hline Conscription age (years) & 18.3 & 0.46 & 18.3 & 0.47 & 18.2 & 0.48 \\
\hline Birth year (year) & 1964 & 7.58 & 1963 & 7.54 & 1964 & 8.06 \\
\hline Mother's age at birth & 27.3 & 5.70 & 28.9 & 5.58 & 29.5 & 4.99 \\
\hline $\mathrm{N}$ & 1,140, & & 19,755 & & 207 & \\
\hline
\end{tabular}


Table 2. The effect of multiple birth on IQ and anthropometric measures in ordinary regression analyses. ${ }^{\mathrm{a}}$

\begin{tabular}{|c|c|c|c|c|}
\hline \multirow[t]{2}{*}{ All men } & \multicolumn{2}{|c|}{$\begin{array}{l}\text { Stratified by parental } \\
\text { socioeconomic position }\end{array}$} & \multicolumn{2}{|c|}{ Stratified by mother's birth year } \\
\hline & Lower SEP & Higher SEP & before 1940 & $r$ after \\
\hline $95 \% \mathrm{CI}$ & $\beta \quad 95 \%$ CI & $\beta \quad 95 \%$ CI & $\beta \quad 95 \%$ CI & $95 \% \mathrm{CI}$ \\
\hline
\end{tabular}

\section{IQ}

Singletons

Twins

0

Triplets

0

$-2.34-2.65,-2.04-2.20$

0

$-2.21-5.58,1.15 \quad-0.50$

$-2.56,-1.84-2.44$

$-4.22,3.21 \quad-2.23$

0.013

$\begin{array}{lll} & 0 & \\ -2.72,-2.15 & -1.87 & -2.27,-1.47 \\ -5.36,0.91 & -0.09 & -4.19,4.00\end{array}$

0

Singletons
Twins
Triplets

p-value of interaction

$-0.50 \quad-0.61,-0.39$

$\begin{array}{lll}0 & 0\end{array}$

$-0.49-1.73,0.75$

$-0.48-0.62,-0.35$

0
-0.54

$-0.72,-0.37$

0
-0.47

$\begin{array}{cc} & 0 \\ -0.60,-0.33 & -0.56 \\ -1.93,1.10 & -0.58 \\ 0.56\end{array}$

$-0.75,-0.37$

$-0.54-2.12,1.05$

$-0.41 \quad-2.37,1.56$

$-0.42$

0.56

\section{$\log$ BMI*100}

Singletons

Twins

Triplets

$$
\begin{array}{ll}
0 & \\
-2.04 & -2.22,-1.85 \\
-3.36 & -5.48,-1.24
\end{array}
$$

p-value of interaction

\section{0}

$-2.34-2.59,-2.10-0$

$-3.99$

$-2.59,-2.10$

$-1.57-1.85,-1.29=-1.96$

$-1.96$

0.0001

$-5.71,0.69 \quad-3.79$
0
$-2.18,-1.74 \quad-2.17$
$-5.96,-1.61 \quad-2.49$
0.42

$-2.50,-1.84$

$-6.83,1.84$

Elbow flexion strength

Singletons

0

Twins

$-11.9-13.2,-10.6$

0

0

0

Triplets

$-20.8-34.2,-7.4$

$-13.6-15.3,-11.9 \quad-9.2$

$-11.2,-7.1$

$-11$.

$-12.7,-9.6 \quad-13.0$

$-13.0-15.2,-10.9$

$\mathrm{p}$-value of interaction

0.01

0.01

$-26.4,23.6$ 
Handgrip strength

Singletons

Twins $\begin{array}{ll}0 & \\ -11.5 & -13.1,-10.0\end{array}$

Triplets $-15.6-30.7,-0.6$

0

0

0

p-value of interaction

$\begin{array}{llll}-13.3 & -15.3,-11.3 & -8.8 & -11.2,-6.4\end{array}$

$\begin{array}{llll}-15.9 & -36.8,4.9 & -15.7 & -37.3,5.9\end{array}$

$-12.9,-9.1$

$-31.4,6.7$

$-12.3$

$-15.0,-9.7$

0.02

$-20.2-44.2,3.9$

Knee extension strength

Singletons 0

Twins

0

Triplets

$-17.5-19.2,-15.7$

0

0

0.39

p-value of interaction

$-19.3-37.5,-1.1$

$-22.9$

$-21.8,-17.3$

$-14.4-17.3,-11.5$

$-16.2$

$-16.9$

0.007

$-15.4 \quad-41.6,10.8$

$\begin{array}{ll}-40.9,7.0 & -22.6\end{array}$

- $3.3,-17.0$

$-40.9,7.0 \quad-22.6 \quad-51.7,6.5$

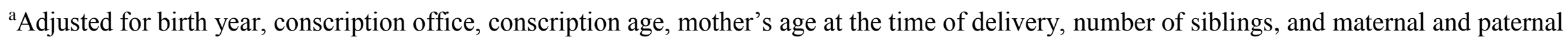
socioeconomic position. 
Table 3. The effect of multiple birth on IQ and anthropometric measures in fixed-effects regression analyses. ${ }^{\text {a }}$

\begin{tabular}{|c|c|c|c|c|}
\hline \multirow[t]{2}{*}{ All men } & $\begin{array}{l}\text { Stratified } \\
\text { socioecono }\end{array}$ & $\begin{array}{l}\text { tal } \\
\text { sition }\end{array}$ & \multicolumn{2}{|c|}{ Stratified by mother's birth year } \\
\hline & Lower SEP & Higher SEP & before 1940 & 1940 or after \\
\hline $95 \% \mathrm{CI}$ & $\beta \quad 95 \% \mathrm{CI}$ & $\beta \quad 95 \% \mathrm{CI}$ & $\beta \quad 95 \% \mathrm{CI}$ & $\beta \quad 95 \% \mathrm{CI}$ \\
\hline
\end{tabular}

\section{IQ}

Singletons

Twins

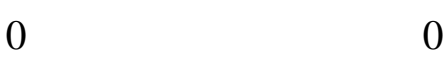

\section{Height}

Singletons $\quad 0$

Twins

$\begin{array}{ll}0 & \\ -1.04 & -1.38,-0.70\end{array}$

$\begin{array}{lll}0 & \\ -0.97 & -1.39-0.54\end{array}$

Triplets

$-0.29-4.52,3.93$

p-value of interaction

$0.24 \quad-5.48,5.95$ 0.81

0
-1.16

$-0.53$

$-1.74,-0.58$

$-6.65,5.58$

0
-0.95

$-0.23$
$-1.33,-0.57 \quad-1.2$
$-5.13,4.67-0.36$
0.56

\section{$\log \mathrm{BMI}^{*} 100$}

Singletons

Twins

Triplets

\section{0}

p-value of interaction

$$
-2.68-3.38,-1.99
$$

$-1.75-10.53,7.03$

\section{0}

$-2.92$

$-0.73$

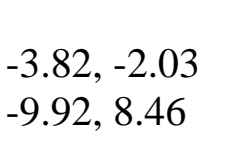

\section{0}

\section{$-2.07$}

$-3.19$

0.58

$\begin{array}{ll} & 0 \\ -3.21,-0.93 & -2.51 \\ -18.76,12.37 & -2.03\end{array}$

$\begin{array}{cc} & 0 \\ -3.29,-1.74 & -3.11 \\ -12.09,8.02 & -1.05 \\ 0.69 & \end{array}$

$-4.58,-1.64$

$-18.26,16.16$

Elbow flexion strength

Singletons

Twins

0

Triplets

$-12.9-18.1,-7.6$

$-18.8-65.3,27.7$

p-value of interaction $\begin{array}{lllll}0 & 0 & 0 & 0\end{array}$

$\begin{array}{lllllll}-14.4 & -21.1,-7.8 & -8.8 & -17.7,0.1 & -12.5 & -18.6,-6.5 & -13.4 \\ -19.2 & -94.4,56.0 & -16.7 & -68.6,35.3 & -15.1 & -73.2,43.0 & -25.1\end{array}$ 0.63
0.78
$-1.93,-0.56$
$-8.53,7.81$ 
Handgrip strength

Singletons 0

Twins

$\begin{array}{lll}0 & 0\end{array}$

Triplets

$$
-13.6-19.6,-7.6
$$

0

$-16.3-23.9,-8.7$

0

p-value of interaction

$-18.8-84.1,46.5$

$-12.1-109.5,85.2$

$-8.9$

$-19.1,1.3 \quad-14.2$

$\begin{array}{ll} & 0 \\ -21.0-7.3 & -12.2\end{array}$

$-12.2-24.3,-0.04$

0.52

0.98

Knee extension strength

Singletons

Twins

$-22.6-29.9,-15.4$

Triplets

0

p-value of interaction

$-44.4-155.2,66.4$

0

$\begin{array}{lllll}-17.4 & -30.1,-4.7 & -21.9 & -30.2,-13.6 & -24.3\end{array}$

$\begin{array}{lllll}-17.4 & -30.1,-4.7 & -21.9 & -30.2,-13.6 & -24.3 \\ -24.5 & -173.3,124.2 & -32.9 & -136.4,70.6 & -41.1\end{array}$

0.67

0.96

$-38.4,-10.3$

$-211.3,129.2$

${ }^{\mathrm{a}}$ Adjusted for birth year, conscription office, and conscription age. 